\title{
DARK ENERGY AND KEY PHYSICAL PARAMETERS OF CLUSTERS OF GALAXIES
}

\author{
Gennady S. Bisnovatyi-Kogan $^{a, b, *}$, Artur Chernin $^{c}$, Marco Merafina $^{d}$ \\ ${ }^{a}$ Space Research Institute Rus. Acad. Sci., Moscow, Russia \\ ${ }^{b}$ Moscow Engineering Physics Institute, Moscow, Russia \\ ${ }^{c}$ Shernberg Astron Inst. MSU, Moscow, Russia \\ ${ }^{d}$ University of Rome "La Sapienza", Department of Physics, Rome, Italy \\ * corresponding author: gkogan@iki.rssi.ru
}

\begin{abstract}
We study physics of clusters of galaxies embedded in the cosmic dark energy background. The equilibrium and stability of polytropic spheres with equation of state of the matter $P=K \rho^{\gamma}$, $\gamma=1+1 / n$, in presence of a non-zero cosmological constant $\Lambda$ is investigated. The equilibrium state exists only for central densities $\rho_{0}$ larger than the critical value $\rho_{\mathrm{c}}$ and there are no static solutions at $\rho_{0}<\rho_{\mathrm{c}}$. At this density the radius of the configuration is equal to the zero-gravity radius, at which the dark matter gravity is balanced by the dark energy antigravity. It is shown, that dark energy reduces the dynamic stability of the configuration. We show that the dynamical effects of dark energy are strong in clusters like the Virgo cluster, which halo radius is close to the zero-gravity radius. It is shown, that the empirical data on clusters like the Virgo cluster or the Coma cluster, are consistent with the assumption that the local density of dark energy on the scale of clusters of galaxies is the same as on the global cosmological scales.
\end{abstract}

KEYWORDS: dark energy, equilibrium models, galaxy clusters.

\section{INTRODUCTION}

Analysis of the observations of distant SN Ia [16, 17] and of the spectrum of fluctuations of the cosmic microwave background radiation (CMB), see e.g. [18], have lead to conclusion that the term, representing "dark energy" (DE) contains about $70 \%$ of the average energy density in the present universe and its properties are very close to the properties of the Einstein cosmological $\Lambda$ term, with a density $\rho_{\Lambda}=\frac{c^{2}}{8 \pi G} \Lambda=0.7 \times 10^{-29} \mathrm{~g} / \mathrm{cm}^{3}$, and pressure $P_{\Lambda}=-\frac{c^{2}}{8 \pi G} \Lambda, P_{\Lambda}=-\rho_{\Lambda}, c=1$. Merafina et al. [15] constructed Newtonian self-gravitating models with a polytropic equation of state in presence of DE. The additional parameter $\beta$ represents the ratio of the density of DE to the matter central density of the configuration. The limiting values $\beta_{\mathrm{c}}$ were found, so that at $\beta>\beta_{\mathrm{c}}$ there are no equilibrium configurations. Dynamic stability of the equilibrium models with DE is analyzed, using an approximate energetic method. It is shown that DE produces a destabilizing effect contrary to the stabilizing influence of the cold dark matter [2, 14].

Local dynamical effects of dark energy were first recognized by Chernin et al. (2000), basing on the studies of the Local Group of galaxies and the expansion outflow of dwarf galaxies around it [1, 5, 7, 12, 19. Chernin et al. 10] have shown that in the nearest rich cluster of galaxies, the Virgo cluster, the matter gravity dominates in the volume of the cluster, while the dark energy antigravity is stronger than the matter gravity in the Virgocentric outflow at the distances of $\simeq 10 \div 30 \mathrm{Mpc}$ from the cluster center. The key physical parameter here is its "zero-gravity radius" which is the distance from the system center, where the matter gravity and the dark energy antigravity exactly balance each other. Bisnovatyi-Kogan and Chernin 4 4 have considered a cluster as a gravitationally bound quasi-spherical configuration of cold non-relativistic collisionless dark and baryonic matter in the cosmological proportion, in presence of a dark energy with the cosmological density $\rho_{\Lambda}$ in the same volume. It was shown that the zero-gravity radius may serve as a natural cut-off radius for the dark matter halo of a cluster. The organization of the paper is the following: in sections 2 and 3 we derive equations, find equilibrium solutions, and analyze a stability of polytropic configurations in presence of a dark energy, in the form of a cosmological constant. The section 4 is devoted to application of these result to the estimation of parameters of Local and Virgo clusters. This presentation follows the papers of Merafina et al. [15, and Bisnovatyi-Kogan and Chernin [4].

\section{MAIn EQUATIONS}

Let us consider spherically symmetric equilibrium configuration in Newtonian gravity, in presence of $\mathrm{DE}$, represented by the cosmological constant $\Lambda$. In this case, the gravitational force $F_{\mathrm{g}}$ which a unit mass undergoes in a spherically symmetric body is written as $F_{\mathrm{g}}=-\frac{G m}{r^{2}}+\frac{\Lambda r}{3}$, where $m=m(r)$ is the mass inside the radius $r$. Its connections with the matter den- 
sity $\rho$ and the equilibrium equation are respectively written as $\frac{\mathrm{d} m}{\mathrm{~d} r}=4 \pi \rho r^{2}, \quad \frac{1}{\rho} \frac{\mathrm{d} P}{\mathrm{~d} r}=-\frac{G m}{r^{2}}+\frac{\Lambda r}{3}$, and the DE density $\rho_{\mathrm{v}}$ is connected with $\Lambda$ as $\rho_{\mathrm{v}}=\frac{\Lambda}{8 \pi G}$. Let us consider a polytropic equation of state $P=K \rho^{\gamma}$, with $\gamma=1+1 / n$. By introducing the nondimensional variables $\xi$ and $\theta_{n}$ so that $r=\alpha \xi$ and $\rho=\rho_{0} \theta_{n}{ }^{n}$, $\alpha^{2}=\frac{(n+1) K}{4 \pi G} \rho_{0}^{\frac{1}{n}-1}$, we obtain the Lane-Emden equation for polytropic models with DE

$$
\frac{1}{\xi^{2}} \frac{\mathrm{d}}{\mathrm{d} \xi}\left(\xi^{2} \frac{\mathrm{d} \theta_{n}}{\mathrm{~d} \xi}\right)=-\theta_{n}^{n}+\beta .
$$

Here $\rho_{0}$ is the matter central density, $\alpha$ is the characteristic radius, $\beta=\Lambda / 4 \pi G \rho_{0}=2 \rho_{\mathrm{v}} / \rho_{0}$ is twice the ratio of the DE density to the central density of the configuration. The spherically symmetric Poisson equation for the gravitational potential $\varphi_{*}$ in presence of $\mathrm{DE}$ is given by

$$
\frac{1}{r^{2}} \frac{\mathrm{d}}{\mathrm{d} r}\left(r^{2} \frac{\mathrm{d} \varphi_{*}}{\mathrm{~d} r}\right)=4 \pi G\left(\rho-2 \rho_{\mathrm{v}}\right), \quad \varphi_{*}=\varphi+\varphi_{\Lambda} .
$$

The gravitational energy of a spherical body $\varepsilon_{\mathrm{g}}$ is $\varepsilon_{\mathrm{g}}=-G \int_{0}^{M} \frac{m}{r} \mathrm{~d} m, \quad m=4 \pi \int_{0}^{r} \rho r^{2} \mathrm{~d} r$, where $M=m(R), R$ is the total radius, and the energy $\varepsilon_{\Lambda}$, representing the interaction of the matter with $\mathrm{DE}$, is given by $\varepsilon_{\Lambda}=\int_{0}^{M} \varphi_{\Lambda} \mathrm{d} m, \quad \varphi_{\Lambda}=-4 \pi G \rho_{\mathrm{v}} r^{2} / 3$. The relations between gravitational $\varepsilon_{\mathrm{g}}$, thermal $\varepsilon_{\mathrm{th}}$ energies, and the energy $\varepsilon_{\Lambda}$ (the virial theorem) have been found by Merafina et al. [15].

$$
\begin{aligned}
& \varepsilon_{\mathrm{g}}=-\frac{3}{5-n} \frac{G M^{2}}{R}-\frac{\Lambda}{2(5-n)} M R^{2}-\frac{2 n+5}{5-n} \varepsilon_{\Lambda}, \\
& \varepsilon_{\mathrm{th}}=\frac{n}{5-n} \frac{G M^{2}}{R}+\frac{n \Lambda}{6(5-n)} M R^{2}+\frac{5 n}{5-n} \varepsilon_{\Lambda} . \\
& \varepsilon_{\mathrm{tot}}=\frac{n-3}{5-n} \frac{G M^{2}}{R}+\frac{(n-3) \Lambda}{6(5-n)} M R^{2}+\frac{2 n}{5-n} \varepsilon_{\Lambda} .
\end{aligned}
$$

\section{EQUILIBRIUM SOLUTIONS}

The equilibrium mass $M_{n}$ for a polytropic configuration which is solution of the Lane-Emden equation is written as

$$
M_{n}=4 \pi\left[\frac{(n+1) K}{4 \pi G}\right]^{3 / 2} \rho_{0}^{\frac{3}{2 n}-\frac{1}{2}} \int_{0}^{\xi_{\text {out }}} \theta_{n}{ }^{n} \xi^{2} \mathrm{~d} \xi .
$$

Using Eq. 1, the integral in the right site may be calculated by partial integration, giving the following relation for the mass of the configuration

$$
M_{n}=4 \pi \rho_{0} \alpha^{3}\left[-\xi_{\text {out }}^{2}\left(\frac{\mathrm{d} \theta_{n}}{\mathrm{~d} \xi}\right)_{\text {out }}+\frac{\beta \xi_{\text {out }}^{3}}{3}\right] .
$$

Here $\theta_{n}(\xi)$ is not a unique function, but depends on the parameter $\beta$, according to Eq. 1. For the limiting configuration, with $\beta=\beta_{\mathrm{c}}$, we have on the outer boundary $\theta_{n}\left(\xi_{\text {out }}\right)=0,\left.\quad \frac{\mathrm{d} \theta_{n}}{\mathrm{~d} \xi}\right|_{\xi_{\text {out }}}=0$, and the mass $M_{n, \text { lim }}$ of the limiting configuration is written as $M_{n, \lim }=\frac{4 \pi}{3} r_{\text {out }}{ }^{3} \beta_{\mathrm{c}} \rho_{0 \mathrm{c}}=\frac{4 \pi}{3} r_{\text {out }}{ }^{3} \bar{\rho}_{\mathrm{c}}$, so that the limiting value $\beta_{\mathrm{c}}$ is exactly equal to the ratio of the average matter density $\bar{\rho}_{\mathrm{c}}$ of the limiting configuration to its central density $\rho_{0 \mathrm{c}}: \beta_{\mathrm{c}}=\bar{\rho}_{\mathrm{c}} / \rho_{0 \mathrm{c}}$. For the Lane-Emden solution with $\beta=0$, we have $\rho_{0} / \bar{\rho}=3.290,5.99,54.18$ for $n=1,1.5,3$, respectively. Let us consider the curve $M\left(\rho_{0}\right)$ for a constant DE density $\rho_{\mathrm{v}}=\Lambda / 8 \pi G$. For plotting this curve in the nondimensional form, we introduce an arbitrary scaling constant $\rho_{\text {ch }}$ and write the mass in the form

$$
M_{n}=4 \pi\left[\frac{(n+1) K}{4 \pi G}\right]^{3 / 2} \rho_{\mathrm{ch}}^{\frac{3}{2 n}-\frac{1}{2}} \hat{M}_{n}
$$

with

$$
\hat{M}_{n}=\hat{\rho}_{0}^{\frac{3}{2 n}-\frac{1}{2}}\left[\frac{\beta \xi_{\text {out }}^{3}}{3}-\xi_{\text {out }}^{2}\left(\frac{\mathrm{d} \theta_{n}}{\mathrm{~d} \xi}\right)_{\text {out }}\right],
$$

where $\hat{\rho}_{0}=\rho_{0} / \rho_{\text {ch }}$ is the nondimensional central density, $\hat{M}_{n}$ is the nondimensional mass. The numerical solutions of the Eq. 1 have been obtained by Merafina et al. [15] for $n=1,3,1.5$. At $n=1$ we have $\xi_{\text {out }}=\pi, 3.490,4.493$, for $\beta=0, \beta=0.5 \beta_{c}=0.089$, $\beta=\beta_{c}=0.178$, respectively. The nondimensional curve $\hat{M}_{n}\left(\hat{\rho}_{0}\right)$, at constant $\rho_{\mathrm{v}}=\beta \rho_{0} / 2$ is plotted in Fig. 1 for $\beta_{\text {in }}=0, \beta_{\text {in }}=0.5 \beta_{c}, \beta_{\text {in }}=\beta_{\mathrm{c}}$, for which $\hat{M}_{1}=\pi, 3.941,5.397$ at $\hat{\rho}_{0}=1, \hat{\rho}_{0} \beta=\beta_{\text {in }}=$ const. At $n=3$ the numerical solution of the equilibrium equation gives $\xi_{\text {out }}=6.897,7.489,9.889$, for $\beta=0$, $\beta=0.5 \beta_{\mathrm{c}}=0.003, \beta=\beta_{\mathrm{c}}=0.006$, respectively. In Fig. 2 we show the behavior of $\left.\hat{M}_{3}\left(\hat{\rho}_{0}\right)\right|_{\Lambda}$, for different values of $\beta_{\text {in }}=0, \beta_{\text {in }}=0.5 \beta_{\mathrm{c}}, \beta_{\text {in }}=\beta_{\mathrm{c}}$, for which $\hat{M}_{3}=2.018,2.060,2.109$, at $\hat{\rho}_{0}=1$, respectively. At $n=1.5$ we have $\xi_{\text {out }}=3.654,3.984,5.086$, for $\beta=0$, $\beta=0.5 \beta_{\mathrm{c}}=0.041, \beta=\beta_{\mathrm{c}}=0.082$, respectively. For $\beta_{\text {in }}=0, \beta_{\text {in }}=0.5 \beta_{\mathrm{c}}, \beta_{\text {in }}=\beta_{\mathrm{c}}$, we have $\hat{M}_{3 / 2}=2.714$, $3.081,3.622$, at $\hat{\rho}_{0}=1$, respectively.

Stability analysis of these configurations done by Merafina et al. [15] using an approximate energetic method [3, 20]. The density in the configuration is distributed according to the Lane-Emden solution at $n=3, \rho=\rho_{0} \theta_{3}^{3}(\xi)$, and we investigate the stability to homologous perturbations. Taking $\rho=\rho_{0} \phi\left(\frac{m}{M}\right)$, with a nondimensional function $\phi$, remaining constant during homologous perturbations we write the derivative of the total energy $\varepsilon$ equal to zero, as an equilibrium equation

$$
\begin{aligned}
& \frac{\partial \varepsilon}{\partial \rho_{0}^{1 / 3}}=3 \rho_{0}^{-4 / 3} \int_{0}^{M} P \frac{\mathrm{d} m}{\phi(m / M)}-0.639 G M^{5 / 3}+ \\
& \quad+0.208 \Lambda M^{5 / 3} \rho_{0}^{-1}-1.84 \frac{G^{2} M^{7 / 3}}{c^{2}} \rho_{0}^{1 / 3}=0 .
\end{aligned}
$$

The dynamical stability is defined by the sign of the second derivative. The DE input in the stability of the configuration is negative like the general relativistic correction [15]. 


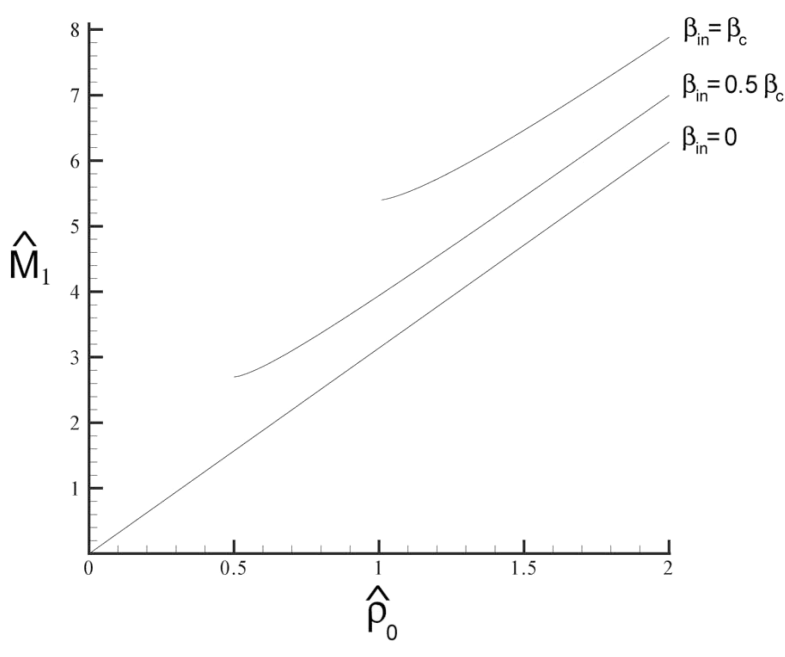

Figure 1. Nondimensional mass $\hat{M}_{1}$ of the equilibrium polytropic configurations at $n=1$ as a function of the nondimensional central density $\hat{\rho}_{0}$, for different values of $\beta_{\text {in }}$. The cosmological constant $\Lambda$ is the same along each curve. The curves at $\beta_{\text {in }} \neq 0$ are limited by the configuration with $\beta=\beta_{\mathrm{c}}$.

\section{Local and Virgo Clusters}

For presently accepted values of the DE density $\rho_{\mathrm{v}}=$ $(0.72 \pm 0.03) \times 10^{-29} \mathrm{~g} / \mathrm{cm}^{3}$, the mass of the local group, including the dark mater input, is between $M_{\mathrm{LC}} \sim$ $3.5 \times 10^{12} M_{\odot}$ [8], and $M_{\mathrm{LC}} \sim 1.3 \times 10^{12} M_{\odot}$ [11]. The radius $R_{\mathrm{LC}}$ of the $\mathrm{LC}$ may be estimated by measuring the velocity dispersion $v_{\mathrm{t}}$ of galaxies in $\mathrm{LC}$ and by the application of the virial theorem, so that $R_{\mathrm{LC}} \sim \sqrt{\left(G M_{\mathrm{LC}} / v_{\mathrm{t}}\right)}$. The estimated $v_{\mathrm{t}}=63 \mathrm{~km} / \mathrm{s}$ is close to the value of the local Hubble constant $H=68 \mathrm{~km} \mathrm{~s}^{-1} \mathrm{Mpc}^{-1}$ 11. The radius of the LC may be estimated as $R_{\mathrm{LC}}=\left(G M_{\mathrm{LC}} / v_{\mathrm{t}}^{2}\right)=(1.5 \div 4) \mathrm{Mpc}$. Chernin et al. [8] identifies the radius $R_{\mathrm{LC}}$ with the radius $R_{\Lambda}$ of the zero-gravity force,

$$
1.2<M_{\mathrm{LC}}<3.7 \times 10^{12} M_{\odot}
$$

and

$$
1.1<R_{\Lambda}<1.6 \mathrm{Mpc} \text {. }
$$

These estimations indicate the importance of DE for the structure and dynamics of the outer parts of LC.

Clusters of galaxies are known as the largest gravitationally bound systems, and the zero-gravity radius is an absolute upper limit for the radial size $R$ of a static cluster with a mass $M$ :

$$
R<R_{\Lambda}=\left[\frac{M}{\frac{8 \pi}{3} \rho_{\Lambda}}\right]^{1 / 3} .
$$

Taking the total mass of the Virgo cluster (dark matter and baryons) $M=(0.6 \div 1.2) \times 10^{15} M_{\odot}$ [4], one finds the zero-gravity radius of the Virgo cluster: $R_{\Lambda}=(9 \div 11) \mathrm{Mpc} \simeq 10 \mathrm{Mpc}$. For the richest clusters like the Coma cluster with the masses $\simeq 10^{16} M_{\odot}$ the zero-gravity radius is about $20 \mathrm{Mpc}$.

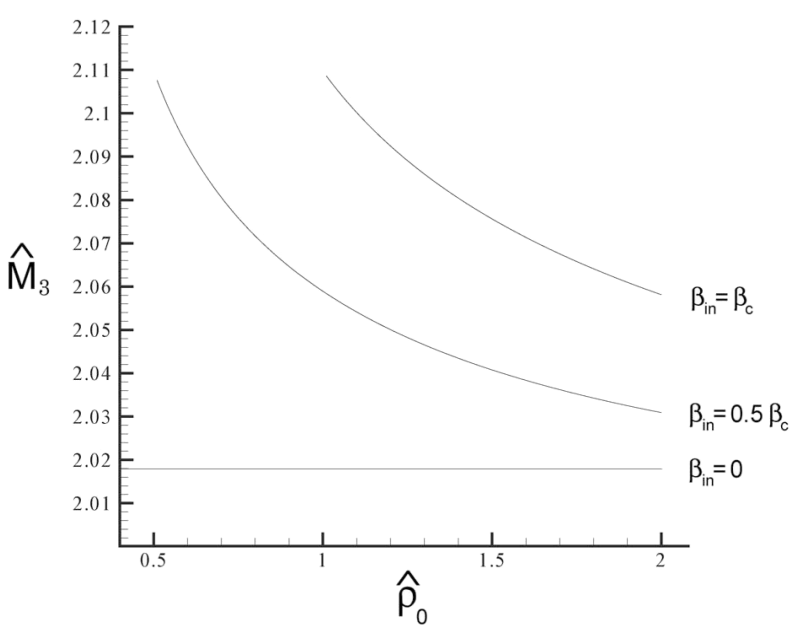

Figure 2. Same as in Fig. 1. for $n=3$.

The data of the Hubble diagram for the Virgo system [13] enable us to obtain another approximate empirical equality:

$$
\left[\frac{R V^{2}}{G M}\right]_{\mathrm{Virgo}} \simeq 1
$$

This relation does not assume either any kind of equilibrium state of the system, or any special relation between the kinetic and potential energies. It assumes only that the system is embedded in the dark energy background and it is gravitationally bound. The data on the Local Group $[8,12$ give

$$
\left[\frac{R V^{2}}{G M}\right]_{\mathrm{Virgo}} \simeq\left[\frac{R V^{2}}{G M}\right]_{\mathrm{LG}} \simeq 1 .
$$

Here we use for the Local Group the following empirical data: $R \simeq 1 \mathrm{Mpc}, M \simeq 10^{12} M_{\odot}$, $V \simeq 70 \mathrm{~km} \mathrm{~s}^{-1}$ [12. Assuming that the the Virgo system has a zero-gravity radius $R_{\Lambda}$, we obtain from the empirical relation that

$$
V^{2} \simeq\left(\frac{8 \pi}{3}\right)^{1 / 3} G M^{2 / 3} \rho_{\Lambda}^{1 / 3} .
$$

The velocity dispersion in the gravitationally bound system depends only on its mass, and the universal dark energy density. The relation Eq. 8 enables one to estimate the matter mass of a cluster by its velocity dispersion

$$
M \simeq G^{-3 / 2}\left[\frac{8 \pi}{3} \rho_{\Lambda}\right]^{-1 / 2} V^{3} \simeq 10^{15}\left[\frac{V}{700 \mathrm{~km} / \mathrm{s}}\right]^{3} M_{\odot} .
$$

The approximate empirical relation may serve as an estimator of the local dark energy density, $\rho_{\text {loc }}$. If the mass of a cluster and its velocity dispersion are independently measured, one has

$$
\rho_{\mathrm{loc}} \simeq \frac{3}{8 \pi G^{3}} M^{-2} V^{6} \simeq \rho_{\Lambda}\left[\frac{M}{10^{15} M_{\odot}}\right]^{-2}\left[\frac{V}{700 \mathrm{~km} / \mathrm{s}}\right]^{6},
$$


what indicates that the observational data on the Local System and the Virgo System provide evidence in favor of the universal value of the dark energy density which is the same on both global and local scales. The gravitational potential $\varphi_{*}(r)$ inside the cluster comes from the Poisson equation Eq. 2

It was found by Bisnovatyi-Kogan \& Chernin 4 in the model of the isothermal halo the maximum of the potential

$$
\varphi_{* \max }=-\frac{3}{2} \frac{G M}{R_{\Lambda}}=-\frac{3}{2} G\left(\frac{8 \pi}{3} \rho_{\Lambda}\right)^{1 / 3} M^{2 / 3} .
$$

The value of $\varphi_{* \max }$ depends on the cluster matter mass $M$ and the universal dark energy density. Its value is the same for any halo profile. It gives a quantitative measure to the deepness of the cluster potential well and determines the characteristic isothermal velocity of the gravitationally bound objects in the cluster,

$$
\begin{aligned}
V_{\text {iso }} & =\left|\varphi_{* \max }\right|^{1 / 2} \\
& =G^{1 / 2}\left(\frac{3}{2}\right)^{1 / 2}\left(\frac{8 \pi}{3} \rho_{\Lambda}\right)^{1 / 6} M^{1 / 3}= \\
& =780\left[\frac{M}{10^{15} M_{\odot}}\right]^{1 / 3} .
\end{aligned}
$$

This velocity is rather close to the mean velocity dispersion, $V \simeq 700 \mathrm{~km} / \mathrm{s}$, of the galaxies in the Virgo cluster; $V_{\text {iso }} \simeq V$ also for the Coma cluster with its matter mass $M \simeq 10^{16} M_{\odot}$ and $V \simeq 1000 \mathrm{~km} / \mathrm{s}$.

The plasma isothermal temperature

$$
\begin{aligned}
T_{\text {iso }} & =\frac{G m}{3 k} V_{\text {iso }}^{2} \\
& =\frac{m}{3 k}\left(\frac{8 \pi}{3} \rho_{\Lambda}\right)^{1 / 3} M^{2 / 3} \\
& =3 \times 10^{7}\left[\frac{M}{10^{15} M_{\odot}}\right]^{2 / 3} \mathrm{~K},
\end{aligned}
$$

This temperature is roughly equal to the temperature of the hot X-ray emitting plasma in clusters like the Virgo cluster or the Coma cluster.

Identifying theoretical value $V_{\text {iso }}$ with the observed value $V$ for typical clusters, we can estimate the matter mass of a cluster, if the velocity dispersion of its galaxies is measured:

$$
\begin{aligned}
M & =\left(\frac{2}{3 G}\right)^{3 / 2}\left(\frac{8 \pi}{3} \rho_{\Lambda}\right)^{-1 / 2} V_{\text {iso }}^{3} \\
& =10^{15} M_{\odot}\left(\frac{V}{780 \mathrm{~km} / \mathrm{s}}\right)^{3} .
\end{aligned}
$$

The relation $M \propto V^{3}$ agrees with the empirical relation following from Eq. 8. In a similar way, the mass may be found, if the theoretical value of the temperature $T_{\text {iso }}$ is identified with the measured temperature of the intracluster plasma:

$$
\begin{aligned}
M & =\left(\frac{3 k}{G m}\right)^{3 / 2}\left(\frac{8 \pi}{3} \rho_{\Lambda}\right)^{-1 / 2} T_{\text {iso }}^{3 / 2} \\
& =\left(\frac{T}{2 \times 10^{7} \mathrm{~K}}\right)^{3 / 2} \times 10^{15} M_{\odot} .
\end{aligned}
$$

If the matter mass of a cluster and its velocity dispersion or its plasma temperature are measured independently, one can estimate the local density of dark energy:

$$
\begin{gathered}
\rho_{\text {loc }}=\rho_{\Lambda}\left(\frac{M}{10^{15} M_{\odot}}\right)^{-2}\left(\frac{V}{780 \mathrm{~km} / \mathrm{s}}\right)^{6}, \\
\rho_{\text {loc }}=\rho_{\Lambda}\left(\frac{M}{10^{15} M_{\odot}}\right)^{-2}\left(\frac{T}{3 \times 10^{7} \mathrm{~K}}\right)^{3} .
\end{gathered}
$$

The empirical data on clusters like the Virgo cluster or the Coma cluster are consistent with our assumption that the local density of dark energy on the scale of clusters of galaxies is the same as on the global cosmological scales.

\section{Conclusions}

(1.) The key physical parameter of cluster of galaxies is the zero-gravity radius

$$
R_{\Lambda}=\left[\frac{M}{\frac{8 \pi}{3} \rho_{\Lambda}}\right]^{1 / 3} .
$$

A bound system must have a radius $R \leq R_{\Lambda}$. For the Virgo cluster $R \simeq R_{\Lambda} \simeq 10 \mathrm{Mpc}$.

(2.) The mean density of cluster's dark matter halo does not depend on the halo density profile and is determined by the dark energy density only: $\langle\rho\rangle=2 \rho_{\Lambda}$.

(3.) The available observational data show that the local density is near the global value $\rho_{\Lambda}$.

\section{ACKNOWLEDGEMENTS}

The work of GSBK was partially supported by RFBR grant 11-02-00602, the RAN program P20 and Grant NSh3458.2010.2. A.C. appreciates a partial support from the RFBR grant 10-02-0178. GSBK is grateful to the organizers of the workshop for support.

\section{REFERENCES}

[1] Baryshev Yu.V., Chernin A.D., Teerikorpi P.: 2001, A\&A, 378, 729

[2] Bisnovatyi-Kogan, G.S.: 1998, ApJ 497, 559

[3] Bisnovatyi-Kogan, G.S.: 2001, Stellar Physics. II. Stellar Structure and Stability (Heidelberg: Springer)

[4] Bisnovatyi-Kogan, G.S., Chernin A.D.: 2012, ApSS, 338,337

[5] Byrd G.G., Chernin A.D., Valtonen M.J.: 2007, Cosmology: Foundations and Frontiers Moscow, URSS

[6] Chernin A.D.: 2001, Physics-Uspekhi, 44, 1099 
[7] Chernin, A.D. 2008, Physics-Uspekhi, 51, 267

[8] Chernin, A.D. et al.: 2009, astro-ph 0902.3871v1

[9] Chernin A.D., Karachentsev I.D., Valtonen M.J. et al.: 2009, A\&A. 507, 1271

[10] Chernin A.D., Karachentsev I.D., Nasonova O.G. et al.: 2010, A\&A. 520, A104

[11] Karachentsev, I.D. et al.: 2006, AJ 131, 1361

[12] Karachentsev I.D., Kashibadze O.G., Makarov D.I. et al.: 2009, MNRAS, 393, 1265

[13] Karachentsev, I.D., Nasonova O.G.: 2010, MNRAS, 405, 1075

[14] McLaughlin, G., Fuller, G.: 1996, ApJ 456, 71
[15] Merafina M., Bisnovatyi-Kogan G.S., Tarasov S.O.: 2012, A\&A, 541, 84

[16] Perlmuter S., Aldering G., Goldhaber G. et al.: 1999, ApJ, 517, 565

[17] Riess A.G., Filippenko A.V., Challis P. et al.: 1998, AJ, 116, 1009

[18] Spergel, D.N. et al. 2003, APJ Suppl., 148, 17

[19] Teerikorpi P., Chernin A.D.: 2010, A\&A 516, 93

[20] Zel'dovich, Ya.B., Novikov, I.D.: 1966,

Physics-Uspekhi 8, 522 